\title{
Elevated Omentin Serum Levels Predict Long-Term Survival in Critically Ill Patients
}

\author{
Mark Luedde, ${ }^{1}$ Fabian Benz, ${ }^{2}$ Jennifer Niedeggen, ${ }^{2}$ Mihael Vucur, ${ }^{2}$ \\ Hans-Joerg Hippe, ${ }^{1}$ Martina E. Spehlmann, ${ }^{1}$ Florian Schueller, ${ }^{1}$ Sven Loosen, ${ }^{1}$ \\ Norbert Frey, ${ }^{1}$ Christian Trautwein, ${ }^{2}$ Alexander Koch, ${ }^{2}$ Tom Luedde, ${ }^{2}$ \\ Frank Tacke, ${ }^{2}$ and Christoph Roderburg ${ }^{2}$ \\ ${ }^{1}$ Department of Internal Medicine III, University Clinic of Schleswig-Holstein, Schittenhelmstrasse 12, 24105 Kiel, Germany \\ ${ }^{2}$ Department of Medicine III, University Hospital RWTH Aachen, Pauwelsstrasse 30, 52074 Aachen, Germany
}

Correspondence should be addressed to Christoph Roderburg; croderburg@ukaachen.de

Received 17 July 2016; Revised 12 September 2016; Accepted 4 October 2016

Academic Editor: Andreas Hillenbrand

Copyright (C) 2016 Mark Luedde et al. This is an open access article distributed under the Creative Commons Attribution License, which permits unrestricted use, distribution, and reproduction in any medium, provided the original work is properly cited.

\begin{abstract}
Introduction. Omentin, a recently described adipokine, was shown to be involved in the pathophysiology of inflammatory and infectious diseases. However, its role in critical illness and sepsis is currently unknown. Materials and Methods. Omentin serum concentrations were measured in 117 ICU-patients ( 84 with septic and 33 with nonseptic disease etiology) admitted to the medical ICU. Results were compared with 50 healthy controls. Results. Omentin serum levels of critically ill patients at admission to the ICU or after 72 hours of ICU treatment were similar compared to healthy controls. Moreover, circulating omentin levels were independent of sepsis and etiology of critical illness. Notably, serum concentrations of omentin could not be linked to concentrations of inflammatory cytokines or routinely used sepsis markers. While serum levels of omentin were not predictive for short term survival during ICU treatment, low omentin concentrations were an independent predictor of patients' overall survival. Omentin levels strongly correlated with that of other adipokines (e.g., leptin receptor or adiponectin), which have also been identified as prognostic markers in critical illness. Conclusions. Although circulating omentin levels did not differ between ICU-patients and controls, elevated omentin levels were predictive for an impaired patients' long term survival.
\end{abstract}

\section{Introduction}

The visceral adipose tissue (VAT) was identified as critical source of bioactive proteins-so-called adipokines-during inflammatory diseases $[1,2]$. By directly regulating hyperglycemia, glucose intolerance, and insulin resistance, adipokines function as prognostic indicators in several diseases [3, 4]. However, in ICU patients, only few data on the regulation, kinetics, and correlation of omentin serum levels with the course of the disease including end points (e.g., death) are available.

Besides well-established adipokines such as leptin or resistin, omentin was recently identified as a new member of the adipokine family [5]. Omentin is encoded by 2 different genes, omentin-1 and omentin-2, and is mainly expressed and secreted by the visceral adipose tissue [5]. In vitro, treatment of human adipocytes with omentin was associated with an enhanced activation of the Akt pathway, leading to an increased glucose uptake upon stimulation with insulin. Moreover, pretreatment with omentin negatively modulated the TNF- $\alpha$ dependent phosphorylation and activation of inflammatory signaling pathways such as p38 or JNK, leading to an impaired production of cytotoxic stress molecules [68]. In vivo, elevated omentin serum concentrations were found in patients with obesity, diabetes, inflammatory bowel diseases, rheumatoid arthritis, asthma, coronary artery disease, and heart failure. Several studies on patients with coronary artery disease even revealed a prognostic function of omentin levels [9-12].

In this study we examined concentrations of circulating omentin in a large cohort of patients from a medical ICU. These data might not only establish omentin as a biomarker 
in critical illness but also help to further define the role of omentin in systemic inflammation and sepsis.

\section{Materials and Methods}

2.1. Study Design and Patient Characteristics. 117 patients that were consecutively admitted between February 2012 and August 2014 to our medical ICU were included in the study. The cohort consisted of 70 males and 47 females, respectively. Patient characteristics are shown in Table 1 . The study protocol was approved by the local ethics committee (Ethics Committee of the University Hospital Aachen, RWTH-University, Aachen, Germany) and conducted in accordance with the ethical standards laid down in the Declaration of Helsinki. Written informed consent was obtained from the patient, his or her spouse, or the appointed legal guardian. Patient information and samples were acquired prospectively, and follow-up was performed as recently described [13]. Presence of septic disease was defined according to the criteria defined in the third consensus definition of sepsis [14]. All other patients were categorized as nonsepsis patients $[15,16]$. Omentin serum levels in critically ill patients were compared with 50 healthy blood donors ( 35 male, 15 female, median age 37 years, and range between 18 and 67) free of chronic diseases like diabetes and cardiac and pulmonary diseases and with normal values for blood counts, C-reactive protein, and liver enzymes.

2.2. Measurements of Omentin Serum Levels by ELISA. Blood collection was performed at the day of admission to the ICU and after 3 days of treatment. Sample handling and analysis of routine laboratory and experimental parameters were performed as described previously $[15,17,18]$. We determined omentin serum levels by using a commercially available enzyme immunoassay (ELISA, Ray Biotech, Norcross, GA, USA) according to manufacturers' instructions.

2.3. Statistical Analysis. Statistics applied in this analysis have recently been described $[15,17,18]$. In summary, data are expressed as median and range. The Mann-Whitney $U$ test and for multiple comparisons the Kruskal-Wallis-ANOVA were used. Correlation analysis was performed by using the Spearman correlation test, and the prognostic value of the variables was tested by univariate and multivariate analysis in the Cox regression model. Kaplan-Meier curves were plotted to display the impact on survival. Finally, ROC curves were generated by plotting sensitivity against 1 - specificity. All statistical analyses were performed with SPSS version 23 (SPSS, Chicago, IL, USA) [19, 20].

\section{Results}

3.1. Omentin Serum Concentrations in Critically Ill and Sepsis Patients. Based on recent data suggesting a potential value of omentin serum levels in inflammatory diseases, we aimed to determine the role of circulating omentin in critically ill patients. Therefore, omentin levels were measured in a large and well defined cohort of 117 ICU patients (Table 1) and in 50 healthy volunteers. Notably, omentin serum levels were
TABLE 1: Study population.

\begin{tabular}{|c|c|}
\hline Parameter & All patients \\
\hline Number & 117 \\
\hline Sex (male/female) & $70 / 47$ \\
\hline Age median (range) [years] & $66(18-90)$ \\
\hline APACHE-II score median (range) & $18(3-40)$ \\
\hline SAPS2 score median (range) & $43(9-80)$ \\
\hline ICU days median (range) & $9(1-137)$ \\
\hline Death during ICU $n(\%)$ & $24.8 \%$ \\
\hline Ventilation time median (range) $[\mathrm{h}]$ & $121(0.0-2966.0)$ \\
\hline Preexisting diabetes $n(\%)$ & $32.8 \%$ \\
\hline HbAlc [\%] & $5.8(4-12.60)$ \\
\hline BMI $\left[\mathrm{kg} / \mathrm{m}^{2}\right]$ & $26.09(15.9-59.5)$ \\
\hline WBC median (range) $\left[\times 10^{3} / \mu \mathrm{L}\right]$ & $12.7(0.1-208)$ \\
\hline CRP median (range) $[\mathrm{mg} / \mathrm{dL}]$ & $116(<5-230)$ \\
\hline Procalcitonin median (range) $[\mu \mathrm{g} / \mathrm{L}]$ & $1.0(0.0-125.2)$ \\
\hline Interleukin-6 median (range) $[\mathrm{pg} / \mathrm{mL}]$ & $73(0-6100)$ \\
\hline Albumin median (range) $[g / d L]$ & $2.8(0.8-4.4)$ \\
\hline INR median (range) & $1.16(0.94-4.62)$ \\
\hline AST median (range) $[U / L]$ & $41(10.0-2591)$ \\
\hline GLDH median (range) $[U / L]$ & $4.15(0.5-720)$ \\
\hline Creatinine median (range) $[\mathrm{mg} / \mathrm{dL}]$ & $1.4(0.1-10.7)$ \\
\hline Urea median (range) $[\mathrm{mg} / \mathrm{dL}]$ & $73(5.0-455)$ \\
\hline Adiponectin median (range) $[\mu g / m L]$ & $10.28(15.98-25.00$ \\
\hline Leptin receptor median (range) $[\mathrm{ng} / \mathrm{mL}]$ & $33.02(5.74-124.46$ \\
\hline Ghrelin median (range) [pmol/L] & $17.4(5-113.8)$ \\
\hline
\end{tabular}

APACHE, Acute Physiology and Chronic Health Evaluation; CRP, C-reactive protein; ICU, intensive care unit; SAPS, simplified acute physiology score; WBC, white blood cell count.

similar between ICU patients and healthy controls (Figure 1(a)). For 79 out of 117 patients, longitudinal measurements were available. Similar to omentin measurements at admission to the ICU, omentin concentrations at day 3 did not differ to healthy controls or to omentin concentrations at day 1 (Figure 1(b)). Accordingly, omentin concentrations were not related to the disease severity (APACHE-2 score at admission to the ICU; Figure 1(c)). Considering the link between circulating omentin and metabolic diseases [21], we further analyzed whether obese patients or patients with type 2 diabetes displayed alterations in their omentin levels. However, at least in our cohort of critically ill patients, levels of circulating omentin were independent of these characteristics (Figures 1(d) and 1(e)). Notably, omentin concentrations were also independent of patient's age or gender (Table 1).

Our cohort of 117 critically ill patients comprises 84 patients with sepsis and 33 patients with nonseptic etiology of disease. No differences in omentin levels between patients with sepsis and those that did not fulfill sepsis criteria became apparent (Figure 2(a)). Moreover, omentin concentrations did not correlate with markers of inflammation or infection such as C-reactive protein (CRP), procalcitonin (PCT), and interleukin-6 (IL-6) (Table 2). We further performed a subgroup analysis to understand whether a deregulation of omentin might be specific for certain disease etiologies. 


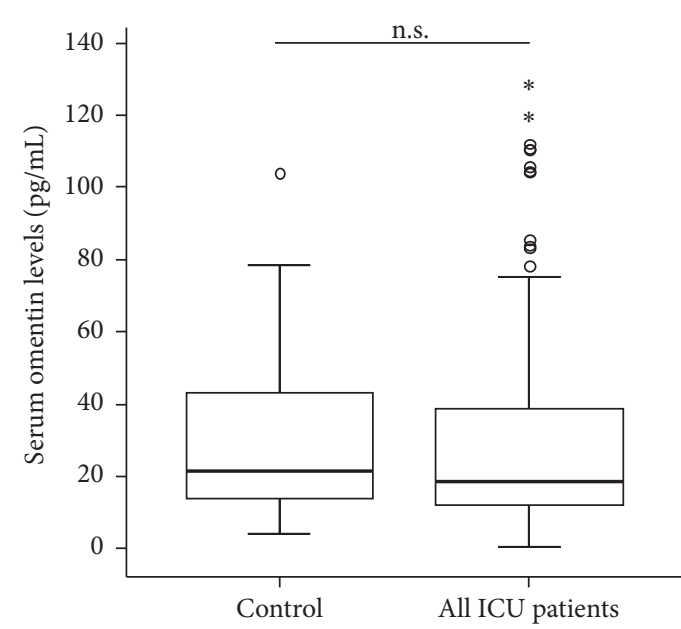

(a)

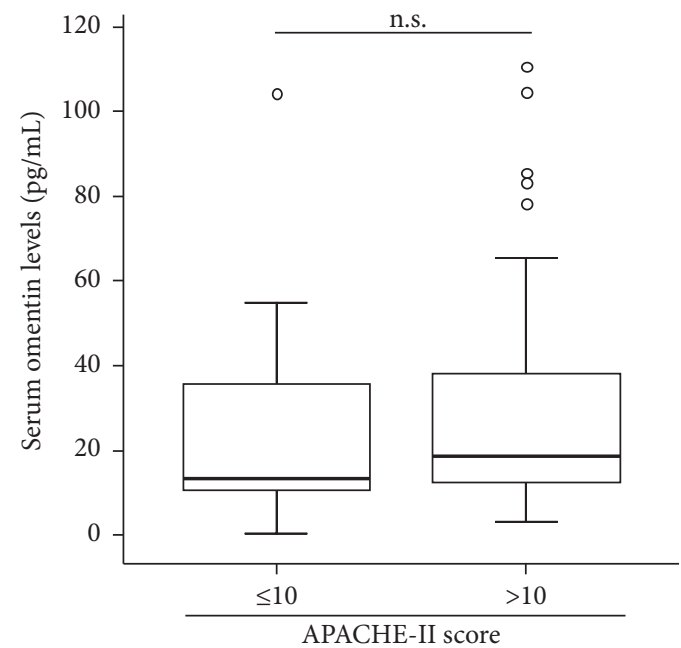

(c)

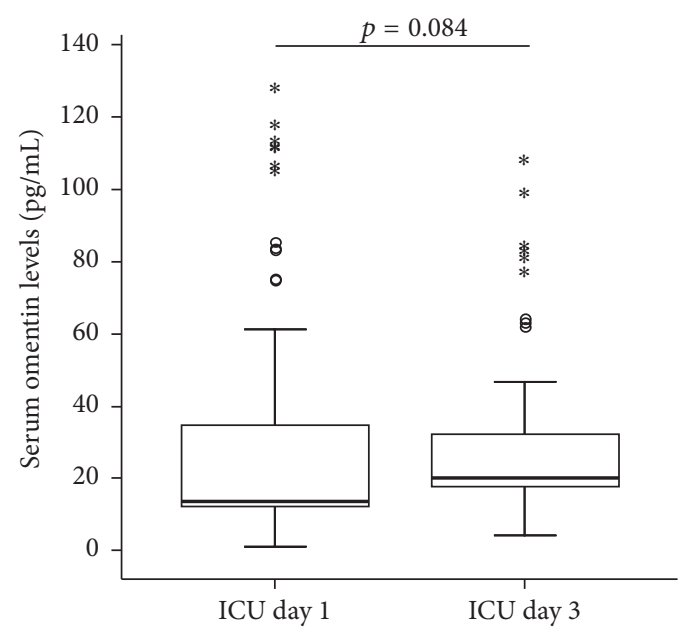

(b)

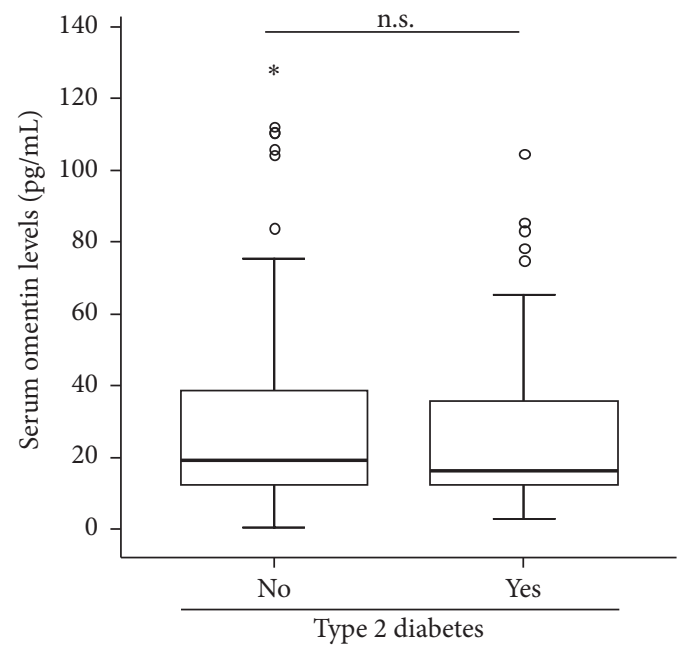

(d)

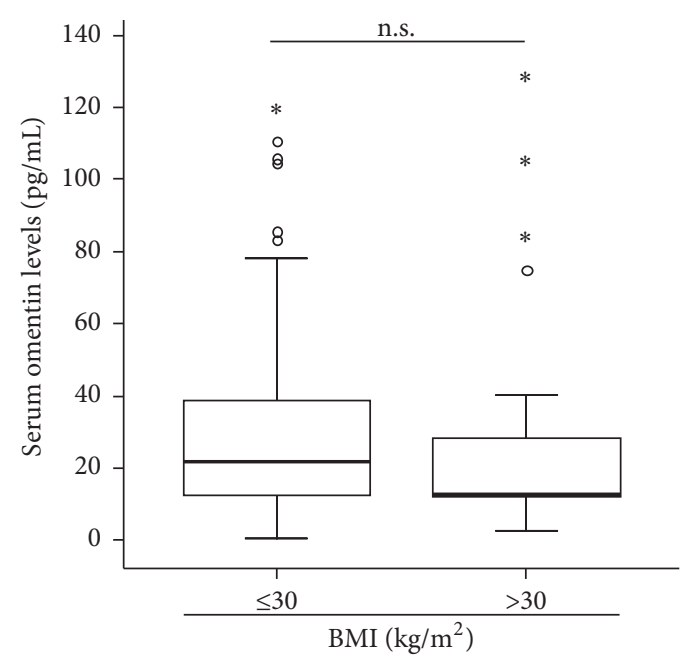

(e)

FIGURE 1: Serum omentin concentrations of critically ill patients at ICU admission and after 3 days of ICU treatment. (a) Omentin levels were determined by ELISA in patients at admission to the ICU $(n=117)$ and in healthy controls $(n=50)$. (b) Omentin levels were measured after three days of ICU treatment $(n=79)$ and compared to levels at admission to the ICU as well as to omentin concentrations of healthy controls. (c) Omentin levels at admission to the ICU were independent of the severity of critical illness (APACHE-2 score at admission to the ICU). (d) Omentin levels were measured in patients with or without type 2 diabetes. (e) Omentin levels were measured in patients with or without obesity. Box plots are displayed, where the bold line indicates the median per group, the box represents $50 \%$ of the values, and horizontal lines show minimum and maximum values of the calculated nonoutlier values; asterisks and open circles indicate outlier values. 


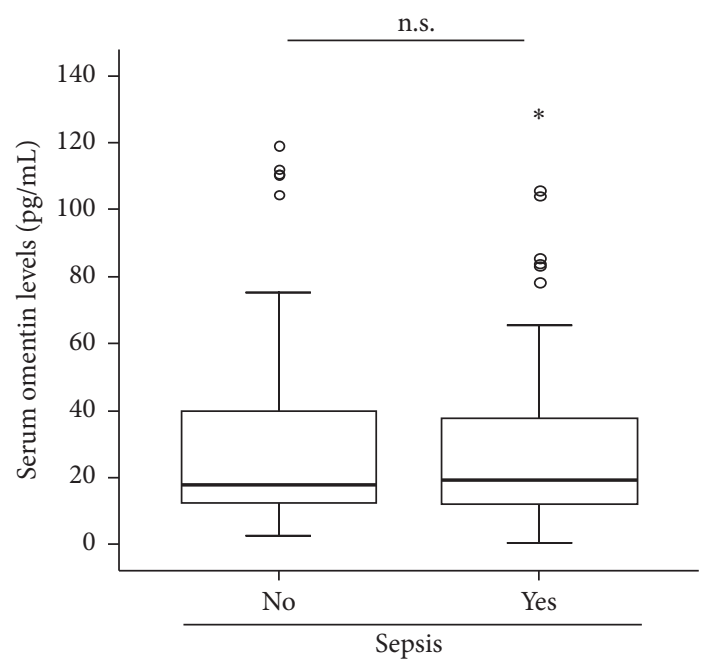

(a)

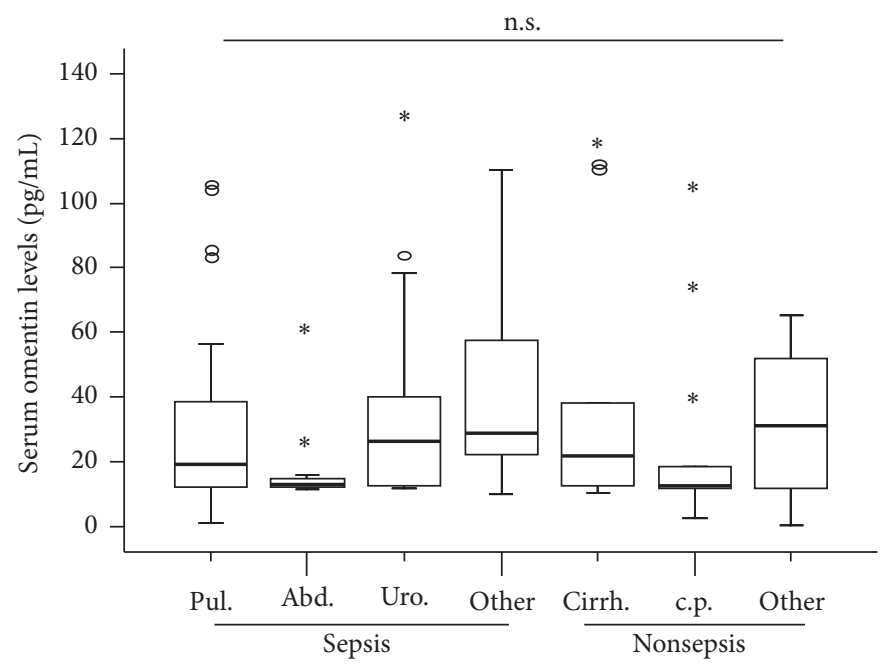

(b)

FIGURE 2: Serum omentin concentrations in patients with sepsis. (a) ICU patients with sepsis $(n=84)$ displayed similar omentin levels compared to patients without sepsis $(n=33, U$ test). (b) Levels of circulating omentin were not different in patients with different etiologies of critical illness. Box plots are displayed, where the bold line indicates the median per group, the box represents $50 \%$ of the values, and horizontal lines show minimum and maximum values of the calculated nonoutlier values; asterisks and open circles indicate outlier values.

TABLE 2: Correlations of omentin serum concentrations at admission day with other laboratory markers at admission to the ICU.

\begin{tabular}{lcc}
\hline Parameter & $r$ & $p$ \\
\hline Markers of liver function & & \\
Albumin & -0.127 & 0.276 \\
INR & 0.136 & 0.147 \\
AST & -0.014 & 0.890 \\
Bilirubin & 0.085 & 0.367 \\
GLDH & -0.103 & 0.317 \\
\hline Markers of inflammation & & \\
CRP & -0.087 & 0.356 \\
Procalcitonin & -0.074 & 0.517 \\
\hline Markers of renal function & & \\
Creatinine & 0.061 & 0.515 \\
Urea & 0.124 & 0.183 \\
\hline Metabolic markers & & \\
Adiponectin & 0.830 & 0.001 \\
Leptin receptor & 0.325 & 0.008 \\
BMI & -0.230 & 0.016 \\
Ghrelin & 0.220 & 0.080 \\
HbAlc & -0.265 & 0.043 \\
Insulin demand d1 & -0.068 & 0.613 \\
Insulin demand d3 & -0.016 & 0.874 \\
C-peptide d1 & 0.229 & 0.081 \\
C-peptide d3 & 0.109 & 0.502 \\
\hline
\end{tabular}

$r$, correlation coefficient; $p, p$ value; $r$ and $p$ values by Spearman rank correlation.

Among the 84 patients with septic disease, 53 were categorized as pulmonary sepsis, 11 as abdominal sepsis, 4 as urogenital sepsis, and 16 as septic diseases with a different/unknown focus. Among the nonseptic patients, 13 suffered from cardiopulmonary diseases, 7 suffered from decompensated liver cirrhosis, and 13 had another etiology of critical illness. By comparing serum omentin concentrations between these different groups we did not observe any differences (Figure 2(b)), thus demonstrating that omentin does not represent a general marker for critical illness and sepsis.

\subsection{Elevated Omentin Concentrations Predict an Increased} Mortality Rate. Several reports suggested an association between elevated omentin concentrations and the probability of survival in patients with inflammatory diseases. To identify an association between circulating omentin and the prognosis of critically ill patients, we compared the omentin levels in survivors and patients that did not survive. In accordance with our previous results, there was no difference between the patients that survived and the patients that died (Figure 3(a)).

Within our cohort of critically ill patients, $25 \%$ died on the ICU and an additional $20 \%$ died during long-term follow-up, median follow-up of 353 days (range 29-800 days). We therefore tested whether omentin levels at admission to ICU were predictive for the patients' long-term prognosis. Strikingly, patients that survived during the long-term follow-up period had significantly lower omentin concentrations than those patients that succumbed to death $(p=0.009$; Figure 3(b)). We next performed Kaplan-Meier curve analysis and Cox regression analysis to determine the impact of elevated omentin levels on patient survival in our cohort of critically ill patients. Interestingly, patients with elevated omentin levels (4th quartile) displayed a significantly higher mortality compared to the other patients (Figure 3(c)). We determined the optimal threshold with the highest Youden Index for omentin levels predicting patient long-term survival. The best 


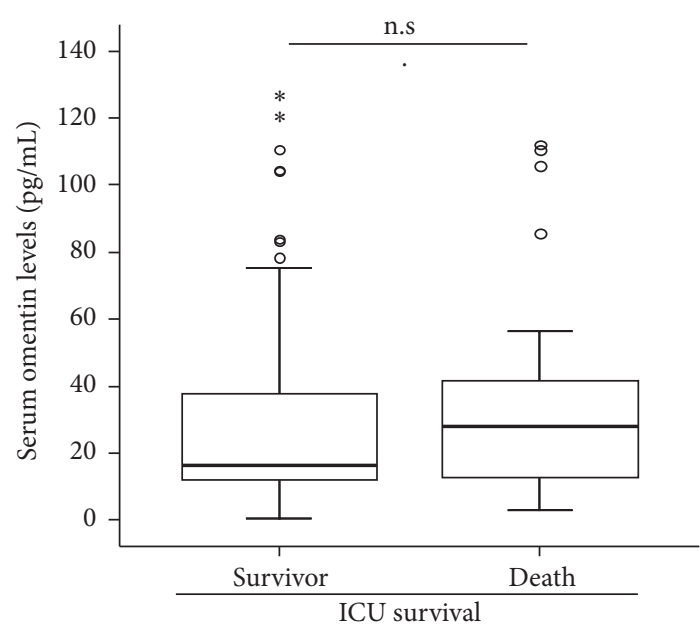

(a)

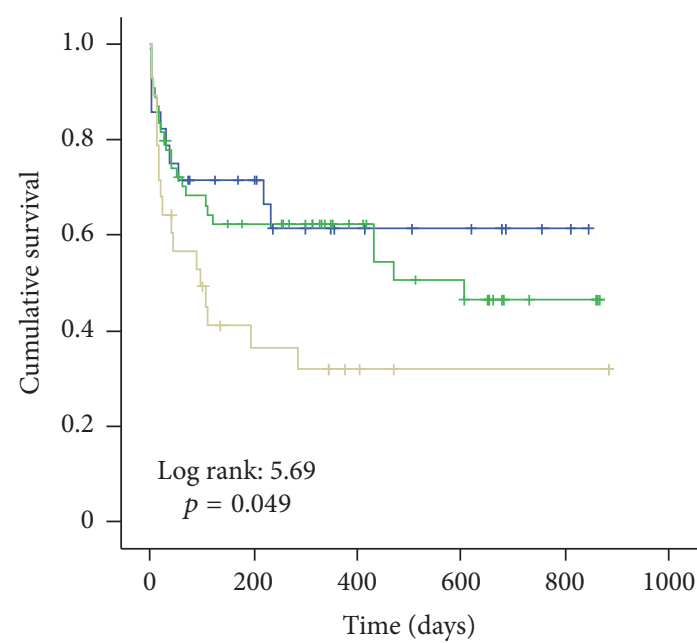

Lower 25\% - Upper 25\%

- 25\%-50\%

(c)

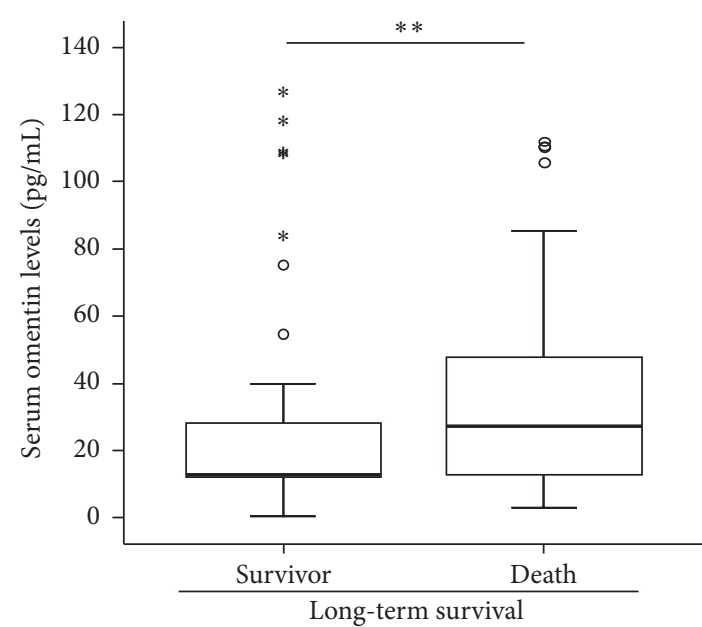

(b)

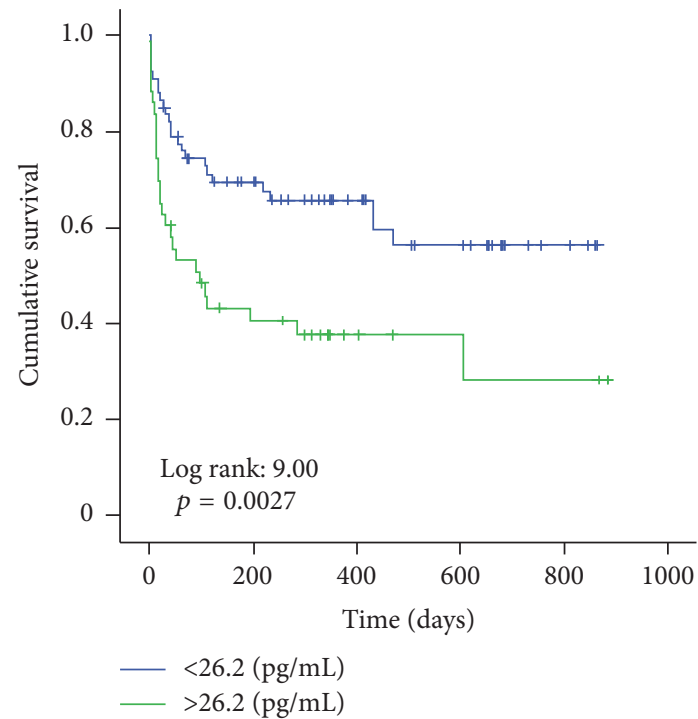

(d)

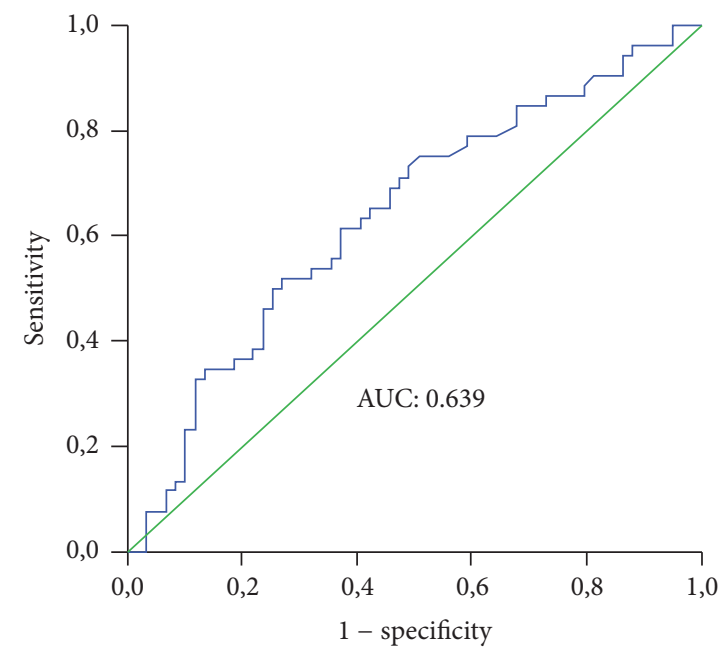

(e)

FIgURE 3: Prediction of long-term mortality by concentrations of circulating omentin. (a) Serum omentin concentrations were measured in patients that died during ICU treatment and survivors. (b) Omentin concentrations analyzed in patients that survived in the long-term followup and patients who did not survive $(U$ test). $(c+d)$ Kaplan-Meier curve analysis with respect to patients' serum omentin concentrations. (e) ROC curve analysis on the value of Omentin measurements for prediction of patients prognosis. Box plots are displayed, where the bold line indicates the median per group, the box represents $50 \%$ of the values, and horizontal lines show minimum and maximum values of the calculated nonoutlier values; asterisks and open circles indicate outlier values. 


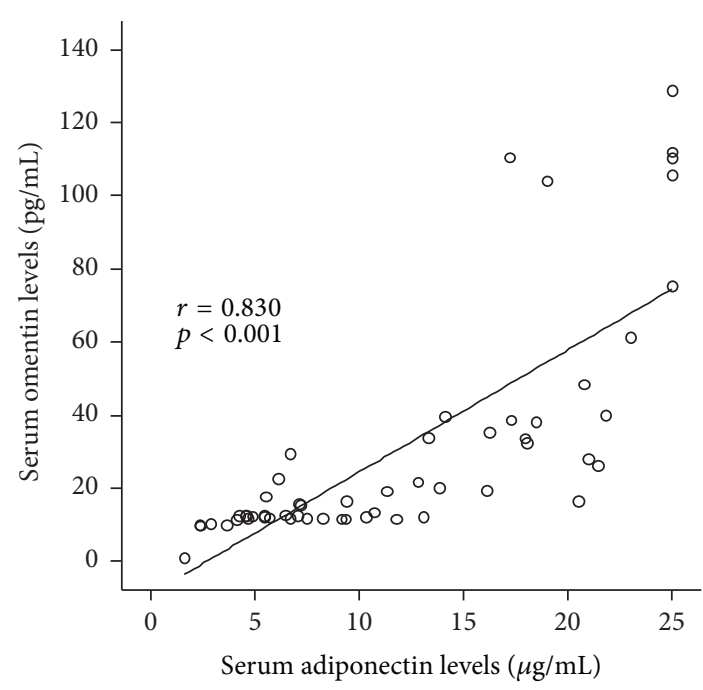

(a)

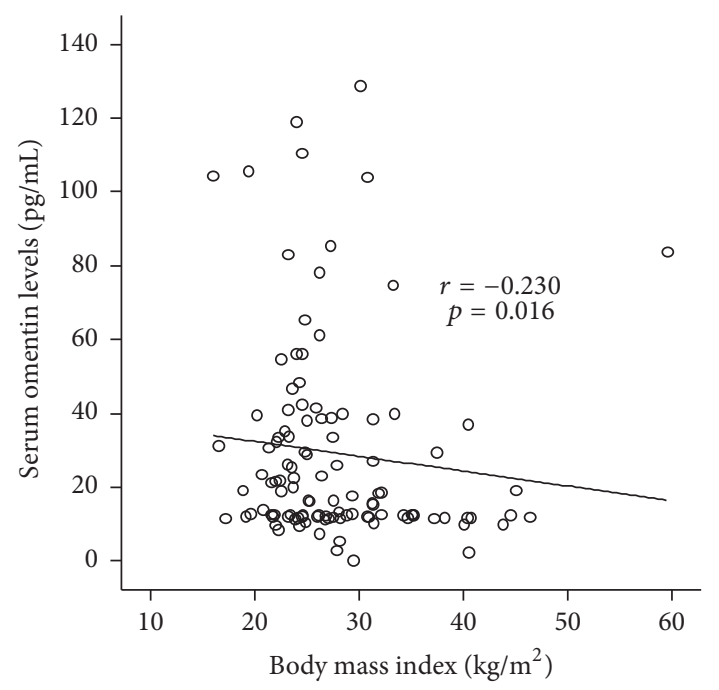

(b)

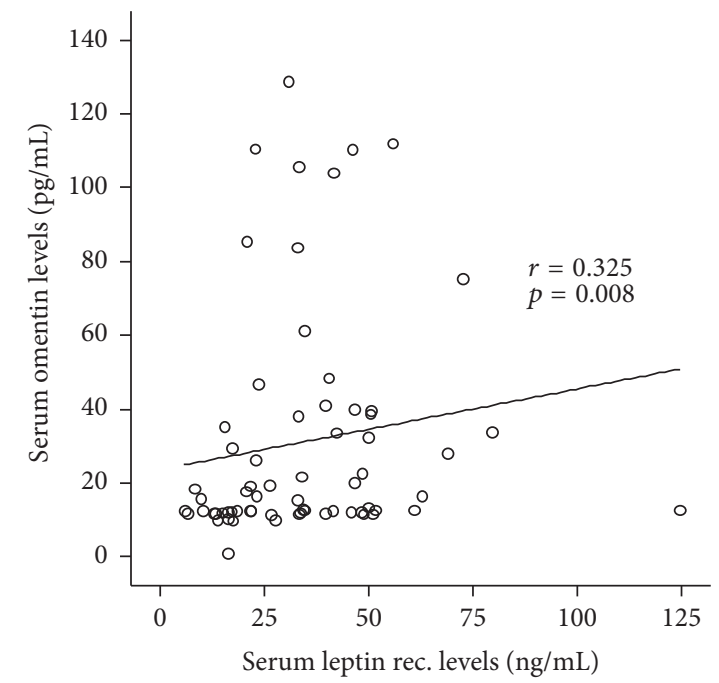

(c)

FIGURE 4: Omentin serum levels are correlated with metabolic parameters of critically ill patients. Serum adiponectin concentrations, body mass index (BMI), and leptin receptor concentrations in critically ill patients are correlated with omentin levels.

sensitivity and specificity scores for survival were obtained when an omentin cutoff level of $26.2 \mathrm{ng} / \mathrm{mL}$ was used. Based on this value, we performed Kaplan-Meier survival analysis, showing significantly impaired survival in critically ill patients with omentin levels $>26.2 \mathrm{pg} / \mathrm{mL}$ compared to patients with lower omentin concentrations (Figure $3(\mathrm{~d})$ ). Of note, the mortality within the "omentin high" group was 63\% compared to $36 \%$ within the "omentin low" group ( $p=0.007$; Pearson Chi-Square test). Finally, multivariate Cox regression analyses identified omentin as an independent prognostic parameter in critically ill patients.

In an attempt to identify factors regulating omentin serum levels in patients with critical illness, we next applied correlation analyses between omentin serum concentrations and a broad set of clinical routine as well as experimental parameters. As mentioned, there was no association between omentin levels and markers for inflammation and infection. Furthermore, in conformity with our data on the lack of an association between omentin and ICU survival, we did not uncover a link between omentin concentrations and markers for organ failure or an unfavorable prognosis on the ICU. Moreover, omentin levels at admission did not correlate with insulin demand or concentrations of C-peptide both at admission to the ICU and after three days of treatment. However, omentin at admission was correlated with adiponectin $(r=0.830, p<0.001)$, leptin receptor $(r=0.325, p=$ $0.008)$, and also the patient's body mass index $((r=0.230$, $p=0.016)$; Figure 4$)$, indicating that metabolically active adipose tissue is the primary source of omentin in critically ill patients.

\section{Discussion}

The traditional concept that scales down adipose tissue to being a storage pool for excess energy has been challenged by multiple studies defining a novel role of adipose tissue as a 
complex hormonally active system that regulates metabolism and inflammation [22]. Cytokines secreted by adipose tissue are called adipokines. Dysregulation of adipokines has been linked to many disease states such as diabetes and obesity [11] and also to cardiovascular diseases [23], bone diseases [24], carcinogenesis [25], chronic kidney disease [26], sepsis [27], and even bipolar disorder [28]. Remarkably, in our cohorts of critically ill patients due to sepsis and different entities like cardiogenic shock and liver failure, adipokines like adiponectin [29], resistin [18], and leptin [4] have been shown to associate with patient survival, underlining a critical role of adipokines in the progression of sepsis and nonseptic critical illness.

Our study determines serum concentrations of omentin in a well characterized cohort of critically ill patients upon admission to the medical ICU. Omentin is a recently identified $\mathrm{Ca}^{2+}$ dependent adipokine that has been denominated as a "good" adipokine, promoting beneficial metabolic effects [30]. Initially described in 2005 [31], it was also referred to as intelectin-1 or "endothelial lectin" [32] due to its close resemblance to lectin in function and structure. It is encoded by two genes, omentin-1 and omentin-2, with omentin-1 being the major circulating form. Thus we here refer to omentin-1 as omentin. This molecule consists of 313 amino acids and is mainly expressed in adipose tissue [30]. With high affinity for galactofuranosyl residues [31], it has been proposed to be in the recognition of specific bacterial components [33].

Omentin expression has been demonstrated to be associated with obesity. While circulating levels of omentin are reduced by obesity [7], weight reduction leads to an increase of omentin concentrations [34]. Likewise, omentin seems to play a protective role in cardiovascular disease [35]. These protective effects of omentin in both obesity and cardiovascular disease seem to be based on disease-specific effects, for example, an insulin sensitizing effect in obesity [36] and modulation of $\mathrm{NO}$ synthase function in the coronary endothelium [37]. On the other hand, omentin seems to exert its effects by interfering with a condition that combines diseases like obesity, diabetes, coronary artery disease, heart failure, pulmonary disease, and chronic kidney disease: a state of chronic low-grade inflammation [30]. Mechanistically, omentin inhibits inflammation via several signaling pathways, for example, by inhibiting tumor necrosis factoralpha (TNF- $\alpha$ ) and NF $\kappa$ B induced expression of intracellular adhesion molecule-1 (ICAM-1) and vascular cell adhesion molecule 1 (VCAM-1) [37]. We analyzed critically ill patients to assess if this comprehensive anti-inflammatory role of omentin might also have a functional role in this collective.

Of note, we did not detect alterations of omentin serum levels in critically ill patients compared to healthy controls. Previous work on serum leptin levels in critically ill patients found an equal effect. Similar to omentin-1, the leptin and also leptin receptor concentrations did not differ between patients with critical illness and healthy controls [4]. Consistent with serum leptin levels, subgroup analyses revealed no significant difference in sepsis and nonsepsis patients. Remarkably, both molecules proved to be an unfavorable indicator of overall cumulative long-term survival, and higher serum levels of omentin were correlated to impaired survival. In contrast to these similarities, both molecules also show important discrepancies in critically ill patients. While leptin serum levels significantly correlated with the incidence of obesity and/or diabetes in these patients, omentin levels did not show a significant correlation with these parameters. In contrast to omentin, serum leptin levels inversely correlated with serum PCT [4]. These opposing results underline the complex regulation and functional differentiation of adipokines. As a possible explanation for these disparate effects, one might speculate that leptin might rather act as a "mediator" of obesity or diabetes related prognostic implications, while omentin-1 exerts effects independent of obesity and diabetes. In this regard, it may be worth mentioning that a negative prognostic impact of obesity has not been conclusively demonstrated in critically ill patients $[38,39]$.

As stated above, a close association of omentin-1 and inflammatory pathways has been proposed [30]. Surprisingly, omentin serum levels did not correlate with markers of acute inflammation or infection like CRP or procalcitonin (PCT). The correlation of these markers with acute critical illness and short-term prognosis has been conclusively demonstrated before $[40,41]$. In this regard, it may seem coherent that omentin serum levels were not differentially regulated in critically ill patients versus healthy controls at ICU admission and did not correlate with short-term prognosis. The fact that high omentin levels correlated with a declined longterm prognosis may shed light on an issue that has not been sufficiently addressed so far: the impact of persistent lowgrade inflammation on long-term prognosis of ICU patients [42]. Inflammation and especially acute systemic inflammatory reaction syndrome (SIRS) have been extensively studied in the acute phase of critical illness, but it is unclear how many patients have evidence of ongoing inflammation in the recovery phase [42]. It is known that the majority of ICU patients exhibit elevated CRP levels at the time of hospital discharge [43]. However, the value of elevated CRP levels as a predictor of ICU outcome is at least unclear [44]. Compared to C-reactive protein, omentin might prove a more promising target in the poorly apprehended setting of post-ICU course of disease, linking chronic inflammation and longterm outcome. Of course, further molecular studies on the complex interactions of omentin and other adipokines during inflammation and critical disease are needed to elucidate their functional role in this context. In this regard, it is still unclear if omentin exerts beneficial effects in critical illness. The highly significant association of elevated omentin serum levels and poor prognosis of ICU patients may reflect either direct malign effects of omentin in critical illness or-more likely - a compensatory upregulation of a generally beneficial [30] molecule. Of note, in line with chronic inflammation, the impact of diabetes [45] and obesity on the outcome of ICU patients is not fully understood. In fact, improved survival of ICU patients with mild to moderate obesity has been proposed, referred to as the obesity paradox in the ICU [39]. Linking obesity and altered metabolism to inflammation, omentin might be a central regulator of the complex network of molecular pathway modulating the course of disease during and after ICU treatment. 
4.1. Limitations of This Study. Our report is not a case control study. Thus, patients and controls are not fully matched regarding sex and age. Moreover, since the panel of clinical information for the control group was not as complete as it was for the patients group, we cannot fully exclude further imbalances between both groups. However, in our analysis, serum omentin levels were not correlated to patients' sex or age; thus it seems unlikely that at least these factors have biased our analysis. Finally, the multiple groups used in analysis of ICU patient subgroups consequently lead to relatively small numbers in each group. Thus, larger studies combining prospective clinical trials and experimental animal models are needed to finally define the role of omentin measurements in a collective of patients with a still unacceptably poor prognosis.

\section{Disclosure}

Mark Luedde and Fabian Benz share first authorship. Tom Luedde, Frank Tacke, and Christoph Roderburg share senior authorship.

\section{Competing Interests}

The authors declare that they have no competing interests related to this manuscript.

\section{Authors' Contributions}

Mark Luedde, Fabian Benz, Jennifer Niedeggen, Mihael Vucur, Hans-Joerg Hippe, Norbert Frey, Christian Trautwein, Tom Luedde, Frank Tacke, and Christoph Roderburg designed the study, analyzed data, and wrote the manuscript. Mark Luedde, Fabian Benz, Mihael Vucur, Jennifer Niedeggen, and Florian Schueller performed measurements. Mark Luedde, Christoph Roderburg, Fabian Benz, Martina E. Spehlmann, and Frank Tacke performed statistical analyses. Alexander Koch and Frank Tacke collected data and organized patient recruitment.

\section{Acknowledgments}

The authors would like to express their gratitude to members of the Luedde Lab, Michaela Roderburg-Goor, and Dr. Jane Beger, for helpful discussions.

\section{References}

[1] I. Ilias, D. A. Vassiliadi, M. Theodorakopoulou et al., "Adipose tissue lipolysis and circulating lipids in acute and subacute critical illness: Effects of shock and treatment," Journal of Critical Care, vol. 29, no. 6, pp. 1130.e5-1130.e9, 2014.

[2] C. Acharya, S. Navina, and V. P. Singh, "Role of pancreatic fat in the outcomes of pancreatitis," Pancreatology, vol. 14, no. 5, pp. 403-408, 2014.

[3] S. J. Van Cromphaut, I. Vanhorebeek, and G. Van den Berghe, "Glucose metabolism and insulin resistance in sepsis," Current Pharmaceutical Design, vol. 14, no. 19, pp. 1887-1899, 2008.

[4] A. Koch, R. Weiskirchen, H. W. Zimmermann, E. Sanson, C. Trautwein, and F. Tacke, "Relevance of serum leptin and leptinreceptor concentrations in critically ill patients," Mediators of Inflammation, vol. 2010, Article ID 473540, 9 pages, 2010.
[5] B. K. Tan, R. Adya, and H. S. Randeva, "Omentin: a novel link between inflammation, diabesity, and cardiovascular disease," Trends in Cardiovascular Medicine, vol. 20, no. 5, pp. 143-148, 2010.

[6] K. Kazama, T. Usui, M. Okada, Y. Hara, and H. Yamawaki, "Omentin plays an anti-inflammatory role through inhibition of TNF- $\alpha$-induced superoxide production in vascular smooth muscle cells," European Journal of Pharmacology, vol. 686, no. 1-3, pp. 116-123, 2012.

[7] C. M. De Souza Batista, R.-Z. Yang, M.-J. Lee et al., "Omentin plasma levels and gene expression are decreased in obesity," Diabetes, vol. 56, no. 6, pp. 1655-1661, 2007.

[8] M. Lapointe, P. Poirier, J. Martin, M. Bastien, A. Auclair, and K. Cianflone, "Omentin changes following bariatric surgery and predictive links with biomarkers for risk of cardiovascular disease," Cardiovascular Diabetology, vol. 13, no. 1, article 124, 2014.

[9] K. Ohashi, R. Shibata, T. Murohara, and N. Ouchi, "Role of antiinflammatory adipokines in obesity-related diseases," Trends in Endocrinology and Metabolism, vol. 25, no. 7, pp. 348-355, 2014.

[10] E. Carmina, "Obesity, adipokines and metabolic syndrome in polycystic ovary syndrome," Frontiers of Hormone Research, vol. 40, pp. 40-50, 2013.

[11] C. Herder, M. Carstensen, and D. M. Ouwens, "Antiinflammatory cytokines and risk of type 2 diabetes," Diabetes, Obesity and Metabolism, vol. 15, no. 3, pp. 39-50, 2013.

[12] J. Yin, P. Hou, Z. Wu, and Y. Nie, "Decreased levels of serum omentin-1 in patients with inflammatory bowel disease," Medical Science Monitor, vol. 21, pp. 118-122, 2015.

[13] C. Roderburg, F. Benz, D. V. Cardenas et al., "Persistently elevated osteopontin serum levels predict mortality in critically ill patients," Critical Care, vol. 19, article 271, 2015.

[14] M. Singer, C. S. Deutschman, C. W. Seymour et al., "The third international consensus definitions for sepsis and septic shock (Sepsis-3)," The Journal of the American Medical Association, vol. 315, no. 8, pp. 801-810, 2016.

[15] F. Tacke, C. Roderburg, F. Benz et al., "Levels of circulating mir133a are elevated in sepsis and predict mortality in critically ill patients," Critical Care Medicine, vol. 42, no. 5, pp. 1096-1104, 2014.

[16] F. Benz, F. Tacke, M. Luedde et al., "Circulating microRNA-223 serum levels do not predict sepsis or survival in patients with critical illness," Disease Markers, vol. 2015, Article ID 384208, 10 pages, 2015.

[17] A. Koch, E. Sanson, A. Helm, S. Voigt, C. Trautwein, and F. Tacke, "Regulation and prognostic relevance of serum ghrelin concentrations in critical illness and sepsis," Critical Care, vol. 14, no. 3, article R94, 2010.

[18] A. Koch, O. A. Gressner, E. Sanson, F. Tacke, and C. Trautwein, "Serum resistin levels in critically ill patients are associated with inflammation, organ dysfunction and metabolism and may predict survival of non-septic patients," Critical Care, vol. 13, no. 3, article R95, 2009.

[19] F. Benz, C. Roderburg, D. Vargas Cardenas et al., "U6 is unsuitable for normalization of serum miRNA levels in patients with sepsis or liver fibrosis," Experimental \& Molecular Medicine, vol. 45, article e42, 2013.

[20] C. Roderburg, F. Benz, D. Vargas Cardenas et al., "Elevated miR122 serum levels are an independent marker of liver injury in inflammatory diseases," Liver International, vol. 35, no. 4, pp. 1172-1184, 2015. 
[21] S. E. Siegelaar, M. Hickmann, J. B. L. Hoekstra, F. Holleman, and J. H. DeVries, "The effect of diabetes on mortality in critically ill patients: a systematic review and meta-analysis," Critical Care, vol. 15, article R205, 2011.

[22] M. K. Badman and J. S. Flier, "The adipocyte as an active participant in energy balance and metabolism," Gastroenterology, vol. 132, no. 6, pp. 2103-2115, 2007.

[23] R. Thoonen, A. G. Hindle, and M. Scherrer-Crosbie, "Brown adipose tissue: the heat is on the heart," American Journal of Physiology-Heart and Circulatory Physiology, vol. 310, no. 11, pp. H1592-H1605, 2016.

[24] E. Neumann, S. Junker, G. Schett, K. Frommer, and U. MüllerLadner, "Adipokines in bone disease," Nature Reviews Rheumatology, vol. 12, no. 5, pp. 296-302, 2016.

[25] C. H. Lee, Y. C. Woo, Y. Wang, C. Y. Yeung, A. Xu, and K. S. L. Lam, "Obesity, adipokines and cancer: an update," Clinical Endocrinology, vol. 83, no. 2, pp. 147-156, 2015.

[26] W. Fenske, T. Athanasiou, L. Harling, C. Drechsler, A. Darzi, and H. Ashrafian, "Obesity-related cardiorenal disease: the benefits of bariatric surgery," Nature Reviews Nephrology, vol. 9, no. 9, pp. 539-551, 2013.

[27] S. P. J. Macdonald, S. F. Stone, C. L. Neil et al., "Sustained elevation of resistin, NGAL and IL-8 are associated with severe sepsis/septic shock in the emergency department," PLoS ONE, vol. 9, no. 10, article el10678, 2014.

[28] B. S. Fernandes, S. Dash, F. Jacka et al., "Leptin in bipolar disorder: a systematic review and meta-analysis," European Psychiatry, vol. 35, pp. 1-7, 2016.

[29] A. Koch, E. Sanson, S. Voigt, A. Helm, C. Trautwein, and F. Tacke, "Serum adiponectin upon admission to the intensive care unit may predict mortality in critically ill patients," Journal of Critical Care, vol. 26, no. 2, pp. 166-174, 2011.

[30] Y.-L. Tan, X.-L. Zheng, and C.-K. Tang, "The protective functions of omentin in cardiovascular diseases," Clinica Chimica Acta, vol. 448, pp. 98-106, 2015.

[31] A. Schäffler, M. Neumeier, H. Herfarth, A. Fürst, J. Schölmerich, and C. Büchler, "Genomic structure of human omentin, a new adipocytokine expressed in omental adipose tissue," Biochimica et Biophysica Acta-Gene Structure and Expression, vol. 1732, no. 1-3, pp. 96-102, 2005.

[32] J.-K. Lee, J. Schnee, M. Pang et al., "Human homologs of the Xenopus oocyte cortical granule lectin XL35," Glycobiology, vol. 11, no. 1, pp. 65-73, 2001.

[33] L. Gerwick, G. Corley-Smith, and C. J. Bayne, "Gene transcript changes in individual rainbow trout livers following an inflammatory stimulus," Fish and Shellfish Immunology, vol. 22, no. 3, pp. 157-171, 2007.

[34] J. M. Moreno-Navarrete, V. Cataln, F. Ortega et al., "Circulating omentin concentration increases after weight loss," Nutrition and Metabolism, vol. 7, article 27, 2010.

[35] V. Katsi, G. Vamvakou, J. Lekakis et al., "Omentin, fat and heart: classical music with new instruments," Heart Lung and Circulation, vol. 23, no. 9, pp. 802-806, 2014.

[36] P. Poirier, T. D. Giles, G. A. Bray et al., "Obesity and cardiovascular disease: pathophysiology, evaluation, and effect of weight loss," Arteriosclerosis, Thrombosis, and Vascular Biology, vol. 26, no. 5, pp. 968-976, 2006.

[37] X. Zhong, X. Li, F. Liu, H. Tan, and D. Shang, "Omentin inhibits TNF- $\alpha$-induced expression of adhesion molecules in endothelial cells via ERK/NF- $\kappa \mathrm{B}$ pathway," Biochemical and Biophysical Research Communications, vol. 425, no. 2, pp. 401406, 2012.
[38] Y. M. Arabi, S. I. Dara, H. M. Tamim et al., "Clinical characteristics, sepsis interventions and outcomes in the obese patients with septic shock: An International Multicenter Cohort Study," Critical Care, vol. 17, no. 2, article R72, 2013.

[39] R. N. Dickerson, “The obesity paradox in the ICU: real or not?" Critical Care, vol. 17, article 154, 2013.

[40] S. M. A. Lobo, F. R. M. Lobo, D. P. Bota et al., "C-reactive protein levels correlate with mortality and organ failure in critically III patients," Chest, vol. 123, no. 6, pp. 2043-2049, 2003.

[41] N. Boussekey, O. Leroy, H. Georges, P. Devos, T. d'Escrivan, and B. Guery, "Diagnostic and prognostic values of admission procalcitonin levels in community-acquired pneumonia in an intensive care unit," Infection, vol. 33, no. 4, pp. 257-263, 2005.

[42] D. M. Griffith, M. E. Vale, C. Campbell, S. Lewis, and T. S. Walsh, "Persistent inflammation and recovery after intensive care: a systematic review," Journal of Critical Care, vol. 33, pp. 192-199, 2016.

[43] T. Akba, S. Karakurt, G. Nlgzel, T. Çelikel, and S. Akalin, “The endocrinologic changes in critically ill chronic obstructive pulmonary disease patients," COPD, vol. 7, no. 4, pp. 240-247, 2010.

[44] S. S. Gülcher, N. A. Bruins, W. P. Kingma, and E. C. Boerma, "Elevated C-reactive protein levels at ICU discharge as a predictor of ICU outcome: a retrospective cohort study," Annals of Intensive Care, vol. 6, article 5, 2016.

[45] J.-L. Vincent, J.-C. Preiser, C. L. Sprung, R. Moreno, and Y. Sakr, "Insulin-treated diabetes is not associated with increased mortality in critically ill patients," Critical Care, vol. 14, no. 1, article R12, 2010. 


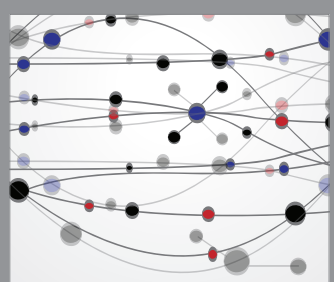

The Scientific World Journal
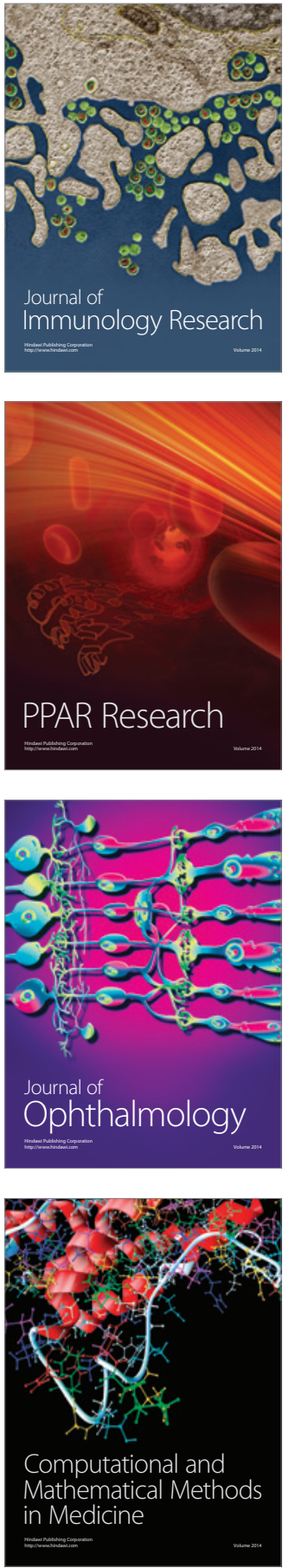

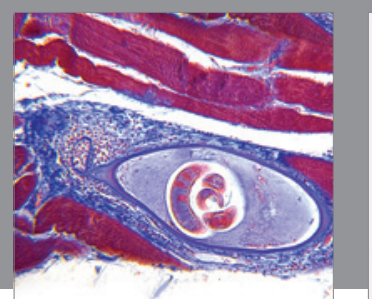

Gastroenterology Research and Practice

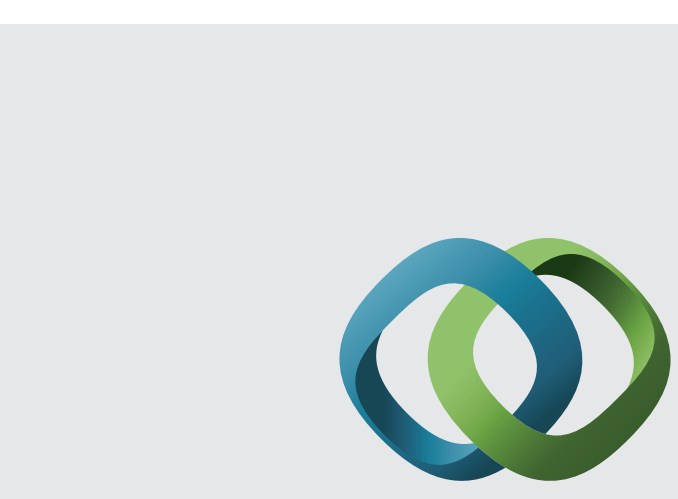

\section{Hindawi}

Submit your manuscripts at

http://www.hindawi.com
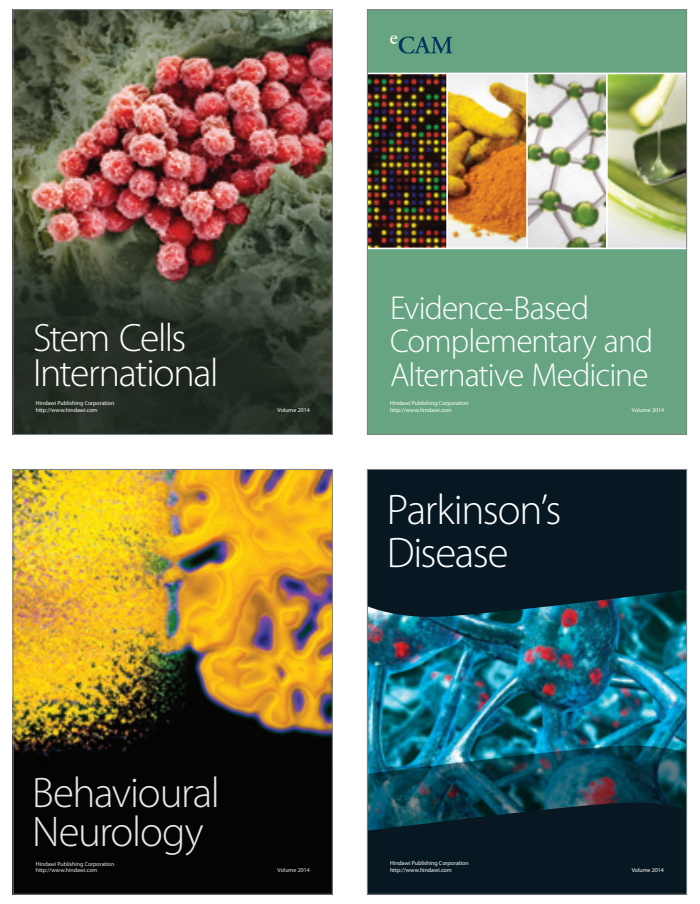
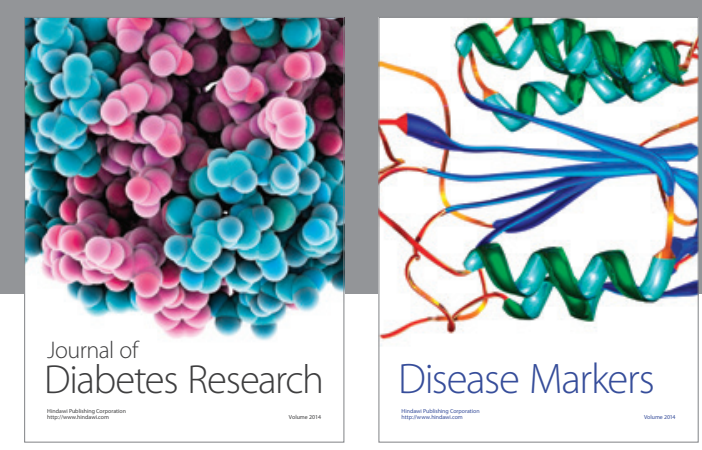

Disease Markers
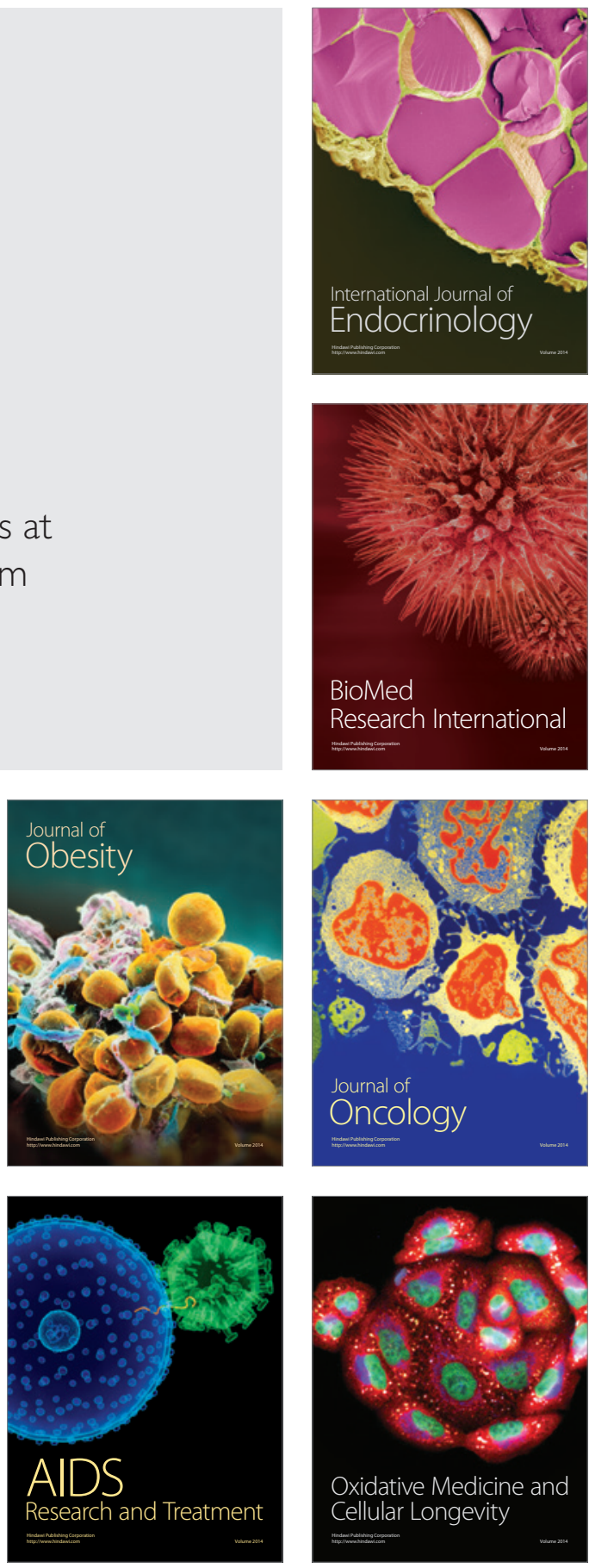